\title{
FARINHA DE CARNE E OSSOS E BUTIRATO DE SÓDIO SOBRE O DESEMPENHO SEMANAL DE FRANGOS DE CORTE
}

Lilian Francisco Arantes de Souza, Melissa de Jesus Bosso Silva, Arthur Benhur Bagnara Rodrigues dos Santos, Giovanna Nascimento Scatolin, Thamires Rodrigues Cavalheiro, Paula Alves de Souza, Jhony Wilher Santana Gilio, Matheus Rocha Ribeiro

Universidade do Oeste Paulista - UNOESTE, Presidente Prudente, SP. E-mail: lilian@unoeste.br

\section{RESUMO}

O objetivo desse trabalho foi avaliar a substituição parcial do farelo de soja por farinha de carne e ossos (FCO) e da suplementação com butirato de sódio (BS) sobre o desempenho de frangos de corte. Foram utilizados 140 pintos distribuídos em DIC em fatorial 2x2 (ausência e presença de BS $x$ ausência e presença de FCO) com 5 repetições. O desempenho foi avaliado semanalmente por meio do consumo de ração (CR), ganho de peso (GP), conversão alimentar (CA) e viabilidade criatória (VC). De 1 a 14 dias e a utilização de FCO reduziu o $\mathrm{CR}$ e o GP, entretanto a inclusão de BS reverteu esses resultados. $A$ inclusão de $B S$ aumentou o CR e o GP de 1 a 7 e 1 a 42 dias. Concluiu-se que tanto a substituição do farelo de soja por FCO como a suplementação com BS são alternativas viáveis na alimentação de frangos de corte.

Palavras-chave: ácidos orgânicos; consumo de ração; conversão alimentar; farinhas de origem animal; ganho de peso.

\section{MEAT AND BONE MEAL AND SODIUM BUTYRATE ON WEEKLY PERFORMANCE OF BROILER}

\begin{abstract}
The aim of this study was to evaluate the partial substitution of soybean meal by meat and bone meal (MBM) and supplementation with sodium butyrate (SB) on the performance of broilers. Were used 140 chick distributed in DIC in factorial 2x2 (absence and presence of BS $x$ absence and presence of FCO) with 5 replications. The performance was evaluated weekly by the feed intake (FI), weight gain (WG), feed conversion (FC) and production viability (PV). From 1 to 14 days and the use of $\mathrm{MBM}$ reduced the $\mathrm{FI}$ and WG, however the inclusion of SB reversed these results. The inclusion of SB increased FI and WG from 1 to 7 and 1 to 42 days. It was concluded that both the substitution of soybean meal by MBM as supplementation with SB are viable alternatives in feeding broilers.

Keywords: animal meal; feed conversion; feed intake; organic acids; weight gain.
\end{abstract}

\section{INTRODUÇÃO}

De acordo com Firman (2006), os produtos de origem animal como fonte de proteína geralmente apresentam custos bastante competitivos em comparação às fontes proteicas vegetais, reduzindo os custos da dieta na maioria das vezes. Geralmente são fonte de proteína de alta qualidade e digestibilidade que podem auxiliar no balanço de aminoácidos exigidos pelos animais, além de excelentes fontes de fósforo disponível e outros minerais. Entretanto, apresentam grande variabilidade em sua composição nutricional (HENDRIKS et al., 2002; MUTUCUMARANA et al., 2015), são potencialmente suscetíveis á oxidação (NOLLET; TOLDRÁ, 2011) e à contaminação microbiana (DAVIES; HINTON, 2000; SAPKOTA et al., 2007).

As preocupações com a presença de microrganismos nas farinhas não se restringem aos danos causados aos animais, mas aos impactos dessas contaminações na saúde humana (SAPKOTA et al., 2007). A utilização de ácidos orgânicos é uma das formas para mitigar a contaminação em farinhas de origem animal (VAN IMMERSEEL et al., 2006). O butirato de sódio (BS) é um sal derivado do ácido butírico produzido a partir da fermentação microbiana de polissacarídeos não amiláceos (LEVY et al., 2015), 
que possui ação bactericida sobre patógenos como Escherichia coli, Salmonella spp, entre outros (VAN DER WIELEN et al., 2000).

$O$ desempenho de frangos de corte pode ser influenciado pela utilização de farinhas de origem animal, bem como pela utilização de ácidos orgânicos. Bozkurt et al. (2004) e Shawle et al. (2010) mostraram que a inclusão de até 5 ou $6,5 \%$ de farinha de carne e ossos (FCO), respectivamente, na dieta de frangos de corte não prejudicou o desempenho dos animais. Por outro lado, Faria Filho et al. (2002) utilizaram dietas com inclusão de 3 ou $6 \%$ de FCO e concluíram que a inclusão piorou o desempenho de frangos de corte. Em relação ao $\mathrm{BS}$, segundo Levy et al. (2015), sua utilização melhorou o ganho de peso e a conversão alimentar de frangos de corte. Ainda, Dolan et al. (2016) obtiveram melhora da conversão alimentar com a utilização de BS.

O objetivo deste trabalho foi avaliar o efeito da substituição parcial do farelo de soja por farinha de carne e ossos e da suplementação com butirato de sódio protegido sobre 0 desempenho semanal de frangos de corte de 1 a 42 dias de idade.

\section{METODOLOGIA}

O experimento foi realizado no Aviário Experimental da UNOESTE. $O$ trabalho foi aprovado pelo Comitê de Ética no Uso de Animais (CEUA) da UNOESTE (Protocolo 565). Foram utilizados 140 pintos da linhagem Cobb, machos, distribuídos em delineamento inteiramente casualizado em esquema fatorial $2 \times 2$ (Butirato de sódio - BS e farinha de carne e ossos - FCO) com 5 repetições de 7 aves cada. Dessa forma, foram utilizados 4 tratamentos ( $T 1=$ sem $B S$ e sem $\mathrm{FCO}$; $\mathrm{T} 2=\mathrm{sem}$ BS e com $\mathrm{FCO}$; $\mathrm{T} 3=\mathrm{com}$ BS e sem FCO; e T4=com BS e com FCO). As aves foram criadas até os 42 dias, recebendo manejo convencional e ração e água ad libitum.

Até 21 dias as aves receberam ração inicial e de 22 a 42 dias receberam ração de crescimento (Tabela 1). As rações foram isocalóricas e isoenergéticas formuladas de acordo com Rostagno et al. (2005). Foi utilizado butirato de sódio microencapsulado (Novyrate C Auster).

O desempenho foi avaliado semanalmente ( 1 a 7 dias, 1 a 14 dias, 1 a 21 dias, 1 a 28 dias, 1 a 35 dias e 1 a 42 dias) por meio do consumo de ração $(C R)$, ganho de peso (GP), conversão alimentar ( $C A=C R / G P)$ e viabilidade criatória (VC=100\%-\%mortalidade).

A análise dos dados foi realizada utilizando o programa estatístico SAS (SAS INSTITUTE, 2001), sendo submetidos à análise de variância e ao teste de Tukey a $5 \%$ de probabilidade $(p<0,05)$. 
Tabela 1. Composição e valores calculados das dietas experimentais da fase inicial (Inic) e de crescimento (Cresc).

\begin{tabular}{lcccccccc}
\hline \multirow{2}{*}{ Ingredientes (\%) } & \multicolumn{3}{c}{ T1 } & \multicolumn{2}{c}{ T2 } & \multicolumn{2}{c}{ T3 } & \multicolumn{2}{c}{ T4 } \\
\cline { 2 - 8 } & Inic & Cresc & Inic & Cresc & Inic & Cresc & Inic & Cresc \\
\hline Milho & 55,94 & 61,56 & 55,78 & 61,49 & 55,94 & 61,56 & 55,78 & 61,49 \\
Farelo de soja & 36,9 & 30,66 & 31,21 & 24,96 & 36,9 & 30,66 & 31,21 & 24,96 \\
Óleo de soja & 3,06 & 4,33 & 3,06 & 4,33 & 3,06 & 4,33 & 3,06 & 4,33 \\
Farinha de carne e ossos & - & - & 5,00 & 5,00 & - & - & 5,00 & 5,00 \\
Fosfato bicálcico & 1,75 & 1,22 & 0,57 & 0,03 & 1,75 & 1,22 & 0,57 & 0,03 \\
Carbonato de cálcio & 0,89 & 0,85 & 0,45 & 0,45 & 0,89 & 0,85 & 0,45 & 0,45 \\
Cloreto de sódio & 0,44 & 0,39 & 0,44 & 0,39 & 0,44 & 0,39 & 0,44 & 0,39 \\
Inerte & 0,26 & 0,30 & 2,69 & 2,63 & 0,16 & 0,225 & 2,59 & 2,555 \\
Premix vitamínico e mineral & 0,24 & 0,20 & 0,24 & 0,20 & 0,24 & 0,20 & 0,24 & 0,20 \\
DL-Metionina & 0,21 & 0,18 & 0,22 & 0,18 & 0,21 & 0,18 & 0,22 & 0,18 \\
L-Lisina & 0,21 & 0,21 & 0,24 & 0,24 & 0,21 & 0,21 & 0,24 & 0,24 \\
Butirato de sódio & - & - & - & - & 0,10 & 0,075 & 0,10 & 0,075 \\
Cloreto de colina & 0,10 & 0,10 & 0,10 & 0,10 & 0,10 & 0,10 & 0,10 & 0,10 \\
\hline & & & & Composição & & & \\
\hline Energia metabolizável (kcal/kg) & 3010 & 3179 & 3010 & 3179 & 3010 & 3179 & 3010 & 3179 \\
Proteína bruta (\%) & 21,40 & 19,00 & 21,40 & 19,00 & 21,40 & 19,00 & 21,40 & 19,00 \\
Cálcio (\%) & 0,87 & 0,71 & 0,86 & 0,71 & 0,87 & 0,71 & 0,86 & 0,71 \\
Fósforo disponível (\%) & 0,55 & 0,33 & 0,55 & 0,33 & 0,55 & 0,33 & 0,55 & 0,33 \\
Sódio (\%) & 0,19 & 0,17 & 0,22 & 0,20 & 0,19 & 0,17 & 0,22 & 0,20 \\
Lisina (\%) & 1,26 & 1,11 & 1,26 & 1,11 & 1,26 & 1,11 & 1,26 & 1,11 \\
Metionina (\%) & 0,50 & 0,44 & 0,50 & 0,44 & 0,50 & 0,44 & 0,50 & 0,44 \\
\hline
\end{tabular}

\section{RESULTADOS}

Houve efeito significativo das dietas sobre CR e GP aos 7 dias (Tabela 2). Aves alimentadas com ração com BS apresentaram aumento no $\mathrm{CR}$ e GP em comparação à ração controle $(p<0,05)$, entretanto, CA e VC não foram influenciadas.
Houve interação significativa $(p<0,05)$ entre BS e FCO sobre CR e GP aos 14 dias (Tabela $3)$.

Aves alimentadas com ração com FCO e sem BS apresentaram menor CR e GP em comparação às demais dietas (Tabelas 4 e 5 ).

Tabela 2. Valores médios para consumo de ração (CR, g), ganho de peso (GP, g), conversão alimentar (CA, $\mathrm{g} / \mathrm{g}$ ) e viabilidade criatória (VC, \%) de frangos de corte de 1 a 7 dias alimentados com dietas sem e com farinha de carne e ossos e butirato de sódio.

\begin{tabular}{lcccc}
\hline 1 a 7 dias & CR & GP & CA & VC \\
\hline Butirato de sódio (BS) & & & & \\
\hline Sem & 172 & 140 & 1,23 & 100,00 \\
Com & 193 & 157 & 1,22 & 100,00 \\
\hline Farinha de Carne e Ossos (FCO) & & & & \\
\hline Sem & 187 & 150 & 1,25 & 100,00 \\
Com & 177 & 148 & 1,20 & 100,00 \\
\hline Probabilidades & & & & \\
\hline BS & 0,0287 & 0,0208 & 0,9655 & - \\
FCO & 0,2826 & 0,7465 & 0,3431 & - \\
BS x FCO & 0,6556 & 0,4659 & 0,8765 & - \\
\hline CV (\%) & 10.59 & 9,85 & 8,81 & 0 \\
\hline
\end{tabular}


Tabela 3. Valores médios para consumo de ração ( $C R, g)$, ganho de peso (GP, g), conversão alimentar (CA, $\mathrm{g} / \mathrm{g}$ ) e viabilidade criatória (VC, \%) de frangos de corte de 1 a 14 dias alimentados com dietas sem e com farinha de carne e ossos e butirato de sódio.

\begin{tabular}{lcccc}
\hline 1 a 14 dias & CR & GP & CA & VC \\
\hline Butirato de sódio (BS) & & & & \\
\hline Sem & 631 & 478 & 1,33 & 97,14 \\
Com & 670 & 518 & 1,29 & 100,00 \\
\hline Farinha de Carne e Ossos (FCO) & & & \\
\hline Sem & 679 & 518 & 1,31 & 97,14 \\
Com & 622 & 476 & 1,31 & 100,00 \\
\hline Probabilidades & & & \\
\hline BS & 0,1082 & 0,0309 & 0,2591 & 0,1220 \\
FCO & 0,0268 & 0,0281 & 0,9863 & 0,1220 \\
BS x FCO & 0,0062 & 0,0156 & 0,5356 & 0,3578 \\
\hline CV (\%) & 7,94 & 7,95 & 4,61 & 3,97 \\
\hline
\end{tabular}

Tabela 4. Desdobramento da interação entre butirato de sódio e farinha de carne e ossos sobre o consumo de ração de frangos de corte de 1 a 14 dias.

\begin{tabular}{lcc}
\hline \multirow{2}{*}{ Butirato de sódio } & \multicolumn{2}{c}{ Farinha de carne e ossos } \\
\cline { 2 - 3 } & Sem & Com \\
\hline Sem & $696 \mathrm{Aa}$ & $566 \mathrm{Bb}$ \\
Com & $662 \mathrm{Aa}$ & $678 \mathrm{Aa}$ \\
\hline
\end{tabular}

* Médias seguidas por letras diferentes, maiúsculas nas colunas e minúsculas nas linhas, diferem entre si pelo teste de Tukey a $5 \%(p<0,05)$.

Aos 21,28 e 35 dias não houve efeito significativo $(p>0,05)$ das dietas sobre nenhuma variável de desempenho das aves (Tabelas 6, 7 e 8).

Tabela 5. Desdobramento da interação entre butirato de sódio e farinha de carne e ossos sobre o ganho de peso de frangos de corte de 1 a 14 dias.

\begin{tabular}{lcc}
\hline \multirow{2}{*}{ Butirato de sódio } & \multicolumn{2}{c}{ Farinha de carne e ossos } \\
\cline { 2 - 3 } & Sem & Com \\
\hline Sem & $521 \mathrm{Aa}$ & $431 \mathrm{Bb}$ \\
Com & $515 \mathrm{Aa}$ & $520 \mathrm{Aa}$ \\
\hline
\end{tabular}

* Médias seguidas por letras diferentes, maiúsculas nas colunas e minúsculas nas linhas, diferem entre si pelo teste de Tukey a $5 \%(p<0,05)$. 
Tabela 6. Valores médios para consumo de ração (CR, g), ganho de peso (GP, g), conversão alimentar (CA, $\mathrm{g} / \mathrm{g}$ ) e viabilidade criatória (VC, \%) de frangos de corte de 1 a 21 dias alimentados com dietas sem e com farinha de carne e ossos e butirato de sódio.

\begin{tabular}{lcccc}
\hline $\mathbf{1}$ a 21 dias & CR & GP & CA & VC \\
\hline Butirato de sódio (BS) & & & & \\
\hline Sem & 1543 & 1085 & 1,42 & 95,71 \\
Com & 1605 & 1140 & 1,40 & 98,57 \\
\hline Farinha de Carne e Ossos (FCO) & & & & \\
\hline Sem & 1582 & 1126 & 1,41 & 95,71 \\
Com & 1566 & 1099 & 1,43 & 98,57 \\
\hline Probabilidades & & & \\
\hline BS & 0,0698 & 0,0743 & 0,4364 & 0,3009 \\
FCO & 0,6261 & 0,3722 & 0,2319 & 0,3009 \\
BS x FCO & 0,1027 & 0,4030 & 0,3158 & 1,0000 \\
\hline CV (\%) & 4,56 & 5,84 & 2,70 & 6,15 \\
\hline
\end{tabular}

Tabela 7. Valores médios para consumo de ração (CR, g), ganho de peso (GP, g), conversão alimentar (CA, $\mathrm{g} / \mathrm{g}$ ) e viabilidade criatória (VC, \%) de frangos de corte de 1 a 28 dias alimentados com dietas sem e com farinha de carne e ossos e butirato de sódio.

\begin{tabular}{lcccc}
\hline $\mathbf{1}$ a 28 dias & CR & GP & CA & VC \\
\hline Butirato de sódio (BS) & & & & \\
\hline Sem & 2834 & 1738 & 1,61 & 94,28 \\
Com & 2784 & 1790 & 1,59 & 98,57 \\
\hline Farinha de Carne e Ossos (FCO) & & & & \\
\hline Sem & 2806 & 1753 & 1,69 & 95,71 \\
Com & 2812 & 1775 & 1,59 & 97,14 \\
\hline Probabilidades & & & \\
\hline BS & 0,5802 & 0,4713 & 0,4002 & 0,1531 \\
FCO & 0,9476 & 0,7664 & 0,4745 & 0,6239 \\
BS x FCO & 0,3746 & 0,2385 & 0,0951 & 0,6239 \\
\hline CV (\%) & 7,12 & 9,08 & 2,97 & 6,63 \\
\hline
\end{tabular}

A Tabela 9 mostra o desempenho das aves aos 42 dias. $O$ uso do BS aumentou CR e GP das aves $(p<0,05)$, sem afetar CA e VC. 
Tabela 8. Valores médios para consumo de ração (CR, g), ganho de peso (GP, g), conversão alimentar (CA, $\mathrm{g} / \mathrm{g}$ ) e viabilidade criatória (VC, \%) de frangos de corte de 1 a 35 dias alimentados com dietas sem e com farinha de carne e ossos e butirato de sódio.

\begin{tabular}{lcccc}
\hline 1 a 35 dias & CR & GP & CA & VC \\
\hline Butirato de sódio (BS) & & & & \\
\hline Sem & 4086 & 2396 & 1,71 & 97,14 \\
Com & 4189 & 2512 & 1,67 & 91,43 \\
\hline Farinha de Carne e Ossos (FCO) & & & & \\
\hline Sem & 4065 & 2430 & 1,67 & 94,28 \\
Com & 4209 & 2478 & 1,70 & 94,28 \\
\hline Probabilidades & & & \\
\hline BS & 0,5783 & 0,2763 & 0,0637 & 0,1635 \\
FCO & 0,4391 & 0,6488 & 0,1913 & 0,9998 \\
BS x FCO & 0,2825 & 0,1414 & 0,2132 & 0,9998 \\
\hline CV (\%) & 9,80 & 9,32 & 3,65 & 9,28 \\
\hline
\end{tabular}

Tabela 9. Valores médios para consumo de ração (CR, g), ganho de peso (GP, g), conversão alimentar (CA, $\mathrm{g} / \mathrm{g}$ ) e viabilidade criatória (VC, \%) de frangos de corte de 1 a 42 dias alimentados com dietas sem e com farinha de carne e ossos e butirato de sódio.

\begin{tabular}{lcccc}
\hline $\mathbf{1}$ a 42 dias & CR & GP & CA & VC \\
\hline Butirato de sódio (BS) & & & & \\
\hline Sem & 4572 & 2372 & 1,93 & 91,43 \\
Com & 5048 & 2604 & 1,94 & 97,14 \\
\hline Farinha de Carne e Ossos (FCO) & & & \\
\hline Sem & 4878 & 2523 & 1,94 & 94,28 \\
Com & 4742 & 2453 & 1,93 & 94,29 \\
\hline Probabilidades & & & \\
\hline BS & 0,0379 & 0,0344 & 0,8385 & 0,1635 \\
FCO & 0,5272 & 0,4980 & 0,9199 & 0,9998 \\
BS x FCO & 0,2735 & 0,0672 & 0,1908 & 0,9998 \\
\hline CV (\%) & 9,78 & 9,00 & 5,71 & 9,28 \\
\hline
\end{tabular}

\section{DISCUSSÃO}

A interação entre as dietas com FCO e BS sobre CR e GP, mostrou que a suplementação apenas com FCO resultou em piores resultados, entretanto, a combinação entre FCO e BS resultou em melhora dessas variáveis. Faria Filho et al. (2002) também observaram menor CR em frangos de corte de 0 a 21 dias alimentados com dietas com inclusão de $\mathrm{FCO}$ de 3 e $6 \%$, além de menor GP nas aves no período de 0 a 49 dias. Por outro lado, Bozkurtet al. (2004) e Shawle et al. (2010) não observaram efeitos da inclusão de $5 \%$ e $6,5 \%$ de FCO, respectivamente, sobre o desempenho das aves. As divergências entre os efeitos da inclusão da FCO sobre o desempenho podem ser atribuídas a variações na composição nutricional das farinhas nos experimentos. Hendriks et al. (2002) mostraram grandes variações nos níveis de proteína bruta, extrato etéreo, matéria mineral e digestibilidade de aminoácidos em diferentes amostras de FCO disponibilizadas comercialmente. Por outro lado, farinhas de origem animal são ingredientes potencialmente sujeitos à contaminação microbiana (DAVIES; HINTON, 2000; SAPKOTA et al., 2007).

Os efeitos benéficos da utilização do BS observados, tanto na interação com a FCO, como isoladamente nas fases de 1 a 7 e 1 a 42 dias, demonstram que esse ácido orgânico pode melhorar o desempenho de frangos de corte. Várias pesquisas corroboram com os resultados 
obtidos, evidenciando melhoras no desempenho de frangos de corte com a utilização de BS (LEVY et al., 2015; CHAMBA et al., 2014; DOLAN et al., 2016). Os benefícios obtidos com a utilização de BS podem ser atribuídos a ação antimicrobiana sobre bactérias patogênicas como Clostridium spp, Escherichia coli e Sallmonella spp (VAN DER WIELEN et al., 2000). As propriedades antimicrobianas do ácido butírico reduzem a colonização entérica por microrganismos patogênicos, equilibrando as substâncias no trato

\section{REFERÊNCIAS}

BOZKURT, M.; BASMACIOGLU, H.; ERGUL, M. Effect of dietary concentration meat and bone meal on broiler chickens performance. International Journal of Poultry Science, v.3, n.11, p.719-723, 2004.

https://doi.org/10.3923/ijps.2004.719.723

CHAMBA, F.; PUYALTO, M.; ORTIZ, A.; TORREALBA, H.; MALLO, J. J.; RIBPOTY, R. Effect of partially protected sodium butyrate on performance, digestive organs, intestinal villi and E. coli development in broilers chickens. International Journal of Poultry Science, v.13 n.7, p.390, 2014.

https://doi.org/10.3923/ijps.2014.390.396

DAVIES, R. H.; HINTON, M. H. Salmonella in animal feed. In: WRAY. C; WRAY. A. Salmonella in domestic animals. New York: CABI, 2000. p. 285300.

https://doi.org/10.1079/9780851992617.0285

DOLAN, L. C.; MORELAND, S.; MORLACCHINI, M.; SPAGNOLI, M.; FUSCONI, G. Performance, safety and tissue residue study for coated sodium butyrate added to broiler feed. International Journal of Poultry Science, v.15, n.5, p. 161, 2016. https://doi.org/10.3923/ijps.2016.161.174

FERNÁNDEZ RUBIO, C.; ORDÓÑES, C.; ABAD GONZÁLEZ, J.; GARCIA GALLEGO, A.; HONRUBIA, M. P.; MALLO, J. J.; BALAÑAFOUCE, R. Butyric acid-based feed additives help protect broiler chickens from Salmonella Enteritidis infection. Poultryscience, v.88 n.5, p.943-948, 2009. https://doi.org/10.3382/ps.2008-00484

FARIA FILHO, D. E.; FARIA, D. E.; JUNQUEIRA, O. M.; RIZZO, M. F.; ARAÚJO, L. F.; ARAÚJO, C. S. S. Avaliação da farinha de carne e ossos na gastrintestinal (FERNÁNDEZ RUBIO et al., 2009), além de efeitos benéficos sobre a mucosa intestinal (CHAMBA et al., 2014).

\section{CONCLUSÃO}

Concluiu-se que a substituição parcial do farelo de soja por FCO piorou o desempenho de frangos apenas na fase de 1 a 14 dias e que a inclusão de BS na dieta melhorou essas variáveis. A inclusão de BS melhorou o desempenho de frangos nas fases de 1 a 7 dias e de 1 a 42 dias.

alimentação de frangos de corte. Revista Brasileira de Ciência Avícola, 2002. https://doi.org/10.1590/S1516635X2002000100005

FIRMAN, J. D. Renderedproducts in poultrynutrition. Essential Rendering, v.125, 2006.

HENDRIKS, W. H.; BUTTS, C. A.; THOMAS, D. V.;JAMES, K. A. C.;MOREL, P. C. A.; VERTEGEN, M. W. A. Nutritional quality and variation of meat and bone meal. Asian Australasian Journal of Animal Sciences, v.15, n.10, p. 1507-1516, 2002. https://doi.org/10.5713/ajas.2002.1507

LEVY, A. W.; KESSLER, J. W.; FULLER, L., WILLIAM,I S.; MATHIS, G. F.; LUMPKINS, B.; VALDEZ, F. Effect of feeding an encapsulated source of butyric acid (ButiPEARL) on the performance of male Cobb broilers reared to $42 \mathrm{~d}$ of age. Poultry Science, v.130, 2015

MUTUCUMARANA, R. K.; RAVINDRAN, V.; RAVINRAN, G.; COWIESON, A. J. Measurement of true ileal phosphorus digestibility in meat and bone meal for broiler chickens. Poultry Science, v.94, n.7, p. 1611-1618, 2015. https://doi.org/10.3382/ps/pev132

NOLLET, L. M.; TOLDRÁ, F. Handbook of analysis of edible animal by-products. Boca Raton (USA): CRC Press, 2011.

ROSTAGNO, H. S.; ALBINO, L. F. T.; DONZELE, J. L.; GOMES, P. C.; OLIVEIRA,R. F.; LOPES, D.C.; FERREIRA, A. S.; BARRETO, S. L. T. Tabelas brasileiras para aves e suínos: composição de alimentos e exigências nutricionais. Viçosa: UFV, 2005. p. 187. 
SAPKOTA, A. R.; LEFFERTS, L. Y.; MCKENZIE, S.; WALKER, P. What do we feed to food-production animals? A review of animal feed ingredients and their potential impacts on human health. Environmental Health Perspectives, 2007. p. 663-670.

https://doi.org/10.1289/ehp.9760

SAS Institute. SAS/STAT ${ }^{\circledR}$ User's Guide (Reléase 8.2). 2001.

SHAWLE, K.; BELIHU, K.; DESSIE, T. The Effect of Replacing Meat and Bone Meal with Soybean Meal on the Performance and Economic Returns of Broiler Chickens. Ethiopian Journal of Animal Production, 2010. p. 95.

VAN DER WIELEN, P. W.; BIESTERVELD, S.; NOTERMANS, S.; HOFSTRA, H.; URLINGS, B. A.; VAN KNAPEN, F. Role of volatile fatty acids in development of the cecalmicroflora in broiler chickens during growth. Applied and
Environmental Microbiology, v. 66, n.6, p.25362540, 2000.

https://doi.org/10.1128/AEM.66.6.2536-

2540.2000

VAN IMMERSEEL, F.; RUSSELL, J. B.; FLYTHE, M. D.; GANTOIS, I.; TIMBERMONT, L.; PASMANS, F.; DUCATELLE, R. The use of organic acids to combat Salmonella in poultry: a mechanistic explanation of the efficacy. Avian Pathology, v.35 n.3, p. 182188, 2006.

https://doi.org/10.1080/03079450600711045

Recebido para publicação em 16/12/2016

Revisado em 18/02/2017

Aceito em 27/03/2017 